\title{
The Analysis of Agent's Knowledge Based on Game Behavior
}

\author{
Jin Sheng GAO 1,a , Chang Le ZHOU 2,3 \\ ${ }^{1}$ Department of philosophy, Xiamen University, Xiamen 361005, China \\ ${ }^{2}$ Department of Cognitive Science and Technology, Xiamen University, Xiamen 361005, China \\ ${ }^{3}$ Fujian Key Lab of the Brain-like Intelligent Systems, Xiamen 361005, China
}

\begin{abstract}
The process of game is actually the process of game behavior which is often accompanied by agent's epistemic process. And Knowledge is a key factor of epistemology. In the past, most of the time game theory confuses information with knowledge. This paper, based on game behavior, analyzes agent's knowledge differences from information. It also analyzes how agent reasons by hidden knowledge behind game behavior, as well as what kind of knowledge is involved in the process of reasoning, and what is the origin of the knowledge. This paper divides the agent's knowledge into two parts: common knowledge and private knowledge, which analyze the specific elements that are corresponding to them in games. In the end, it constructs a knowledge model of game agent, furthermore, by this model, the essence of knowledge held by agents in their game would be presented.
\end{abstract}

\section{Introduction}

In recent years, many logicians, economists and computer experts have been studying games using epistemic logic. Some researchers have studied the assignment games [1], some like to study games using logic techniques [2], such as the preference in strategy choice [3], the dynamic logic in extended games[4].As further research goes, the boundary between logic and games is getting increasingly blurring.In game behavior, according to the logical points of view, the reasoning involved in games can be divided into deductive reasoning and inductive reasoning.But both deductive reasoning and inductive reasoning will involve agent's cognitive behavior in the process of interaction with each other, i.e., the agent's choicesin strategy game are based on their common knowledge and background knowledge.In particular, common knowledge has a direct impact on the game agent's behavior.More topics involved are the relationships between common knowledge and agent's background knowledge, how they transform from each other, and the properties of knowledge in game behavior, which will be discussed and explored in this paper.

This paper consists of five sections.In section 2 , we introduce the general expression of knowledge. In this section we define the concept of knowledge by its history and development. In section 3, it analyzes the types of agent's knowledge in game behavior. In section 4, we explain the process of knowledge formation and the structure of knowledge. In this section, we differentiate knowledge with data, information, and so on. In the end section, we discuss the results and of this paper and the future work.

\section{The general expression of knowledge}

Knowledge is a terminology that is frequently defined by many academic disciplines. There are a great of philosophers in epistemology had researched it.They have their own different ideas about knowledge. About the definition of the knowledge, they have been to debate endlessly. Here comes to a basic question: what is knowledge? For this, the most classic should be traced back to Plato directly. For example, his article "Theaetetus" is one of the most influential works about knowledge. In his book, he argues, knowledge is a true belief, which satisfies the following three conditions: The agent knows $\varphi$, when only if (i) $\varphi$ is true, (ii) agent believes $\varphi$, (iii) agent hasdefinite evidences to believe $\varphi$.

Therefore, Plato defines knowledge as something that is justified, true, andbelieved. However, this traditional definition had been challenged byGettier. In "Is justified true belief knowledge?",Gettier puts forward some counterexamples on traditional definition. In his cases, even though these conditions are all satisfied, agent still doesn't know $\varphi$ [5].In other words, these conditions actually cannot explain what knowledge is.Because we can hold a true belief that can be distinguished, but we believe that this belief is true just with some sort of accident. And then this belief is not as knowledge.

After Gatthier case, whether need to modify the initial conditions, or whether to introduce other conditions?In Epistemology, for example, some advocates have presented different ways based on Gattier case, and then

\footnotetext{
${ }^{\mathrm{a}}$ Corresponding author: Hi.Jackson@163.com
} 
there are forming fundamentalism,conheretism and contextualism, and so on[6].

Various suggestions have been proposed, but none of them got the consensus, and the question remains. In the current, the amendments of the knowledge definition or a new definition haven't arrived at a consensus. Obviously, Plato's ancient definition of knowledge has a profound impact on. Therefore, Plato's ancient definition of knowledge has been recognized by most people.

Based on the specific property of game behavior, and combine with the above analysis, we have the following definition about knowledge:

Definition 1(knowledge):Knowledge is a kind of cognitive stateincludingconnotative state (such as practical skills or expertise) and explicit state (such as disciplinary theory), which agent holds after through his experienceand education.

In fact, the factors of knowledge and the relations among them can be showed by figure 1 as follows:

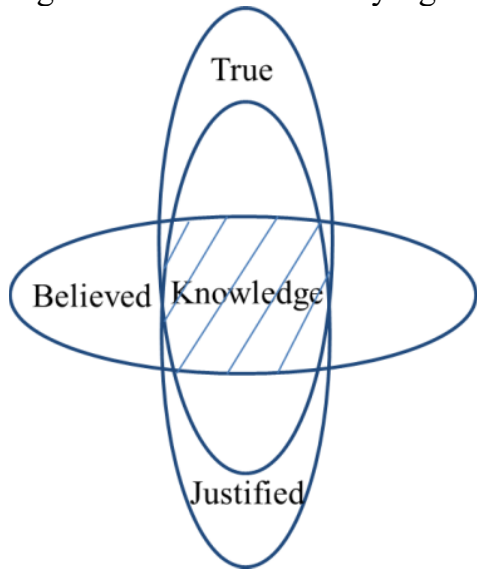

Figure 1. The concept of knowledge

\section{Knowledge types of behavioral agent}

Just as the above-mentioned definition of knowledge, game behavior involves agent cognitive, and agent cognitive involves external information, agent's own background, as well as the implied knowledge deduced by the agent through external information and its own background knowledge, et al. From the perspective of epistemology, knowledge has a variety of types; accordingly, for each specific discipline area, knowledge can be divided into corresponding types. For the game behavior, the knowledge of game agents can be divided into two categories: common knowledge and private knowledge according to these agents' situation.

\subsection{Common knowledge of agents}

The notation of common knowledge is first proposed by the philosopher, Lewis. He mentioned the general idea of common knowledge in his book "Conventions: A Philosophical Study" in 1969: It's a knowledge that everyone knows and everyone knows other knows and ... recursively [7]. Though the common knowledge is first proposed by Lewis, Aumann firstly gave out the formal definition of common knowledge at 1976 [8]. This definition is aimed at game theory and can eliminate infinite recursions, such that people is accustomed to regard Aumann as the main contributor for the research of common knowledge.

Nowadays, there are two expressions of "Common Knowledge", the former introduce modal operators in epistemic logic into the definition, and the later one is a kind of Aumann's set structure expression.

\subsubsection{Modal operator expression}

To characterize the properties of "Common Knowledge", a corresponding formal system $S_{C K}$ is usually constructed in epistemic logic. Similar to other epistemic logic systems, $S_{C K}$ is also an extension of propositional logic. The extended parts include: a cognitive community $N$, modal operators $K_{i}(i=1,2,3, \cdots, \mathrm{n})$, modal operator $K_{S}$ $(S \subseteq N)$, modal operator $E_{N}$ and the operator $C$, where $K_{i} \varphi$ means "Agent $i$ knows $\varphi$ ", $E_{N} \varphi$ means "Each member in community $N$ knows", $K_{S} \varphi$ means "Some members in $N$ know", $C \varphi$ means " $\varphi$ is Common knowledge for everyone".In addition, following axioms are added to the classical logic system [9]:

$$
\begin{aligned}
& K_{S} \varphi \equiv \bigvee_{i \in N} K_{i} \varphi \\
& E_{N} \varphi \equiv \bigwedge_{i \in N} K_{i} \varphi(2) \\
& C \varphi \equiv \bigwedge_{i=1}^{n} E^{n} \varphi(n=1,2,3, \cdots)
\end{aligned}
$$

$N$ can be infinite in theory, but the language of epistemic logic is usually finite.

Then, we usualy use Kripke model to define the above syntax structure, such thatwith semantic meanings.Given a cognitive model $\left\langle S, R_{i}, V\right\rangle$, where

(i) $S$ is a non-empty of cognitive states, $s_{m} \in S$;

(ii) $R_{i}$ is an accessible relation between possible worlds, $(i=1,2,3, \cdots, \mathrm{n})$;

(iii) $V$ is a truth assignment for each proposition in possible worlds.

In this way, the semantics of modal operators can be determined: by definition, $K_{i} \varphi$ is true at cognitive state $s_{0}$, if and only if $\varphi$ is true in $s_{i} \in S$ where $s_{0} R_{i} s_{m}$.

\subsubsection{Aumman'sstructure expression}

The Nobel economics laureate,Aumann, utilize set theory to define common knowledge in 1976. Let $S$ be the set of all states and event $E$ be the set of all subsets of $S$. For each member $i(i=1,2,3, \cdots, \mathrm{n})$, an information set is defined upon $S$ and $P_{i}$. This information set represents the cognitive state of the member in this state. At state $s_{0}$, that the statement "member $i$ knows" obtains a state from $P_{i}\left(s_{m}\right)$, but the obtained state is explicitly unknown. This fully embodies the essence of dynamic game under incomplete information. In such a dynamic game scene, agents can have several decision nodes in some information set in the game tree, but the agents can't tell which decision node they are located in, thus these decision nodes belong to the same information set. Now, we will define a knowledge function $K_{i}$ by the following way [10]: 


$$
K_{i} \varphi \equiv\left\{s_{m} \in S \mid P_{i}\left(s_{m}\right) \subset \varphi\right\}
$$

$K_{i} \varphi$ is the set of all states that $i$ knows the event $\varphi$ can have. Similar to the above modal logic formulization, we can define a operator to represent "Everyone knows $\varphi$ ":

$$
E_{i} \varphi \equiv \cap_{i=1}^{N} K_{i} \varphi
$$

Similarly, we can define the common knowledge in Aumann's way as follows:

$$
C \varphi \equiv \cap_{n=1}^{\infty} E^{n} \varphi
$$

The Aumman's structure expression can be transformed to Kripkemodel $<S, P_{i}>$, where the state set $S$ is similar to cognitive states and the information set $P_{i}$ defines the accessible relation between states.

\subsubsection{Common knowledge in game behavior}

AfterAumann's definition of common knowledge, game theorists always like to use something that similar to "someone knows what the other agent knows" as common knowledge. However, many game theorists are unwilling to define the form of common knowledge on the structure of game theory. There are some technical problems as it is necessary to adjust the previous research results in many ways. So they are used to stopping in the form of common knowledge. Because of the different research perspective, the common knowledge involves different elements. For the game behavior, the common knowledge is as follows:

(I) First of all, "the hypothesis of rational man" is the common knowledge. Rationality is as the most basic conditions for game occurrence and strategy selection. In game theory, behavioral agent is a rational person who will maximize the pursuit points of their own income. However, in epistemic logic, it is usually used to indicate the best response of the player who knows or believes certain knowledge. The best response, while, is a kind of preference logic, specifically to characterize the optimal reaction function of action logic. In this regard, it can be subdivided into "weak rationality" and "strong rationality":

Definition 2 (Weak Rationality, Strong Rationality): Weak rationality means behavioral agent is not stupid and will always choose the optimal strategy in his cognitive range to maximize his payoff, but the agent may not know the chosen strategy is strictly dominant. Strong rationality refers to the agent can find a complete measure in overall strategy, and select out a strictly dominant.

(II) Secondly, the structure of the game is a common knowledge. Basic elements of a game includes: the behavioral agent, the rules, the outcome, and the payoff [11]. The structural elements of the game must become common knowledge among the agents. For example, in the dynamic game of incomplete information, game players must recognize the game order between them.

(III) In addition, the characteristic elements of different games are common knowledge. Since the game can be divided into different types and each type of game has different characteristic elements, which become common knowledge among the agents and take play game behavior. For example, for the incomplete information game structure, although the game player $i$ don't clear the opponent's type $P_{-i}$, player $i$ must be clear his own type of belief $P_{i}$ and the function $A_{i}\left(T_{i}\right)$ mapping types space $T_{i}$ to action space $A_{i}$. All these knowledge must be the common knowledge between game players.

\subsection{Private knowledge of agents}

Game agent's knowledge is not just common knowledge, there is corresponding with the private knowledge. Specifically, private knowledge mainly embodied in the following aspects:

(I) Formulti-stage dynamic game with complete information and static game, the game agent cannot obtain the competitor's accurate action space. (II) The opponent type $P_{-i}$. Game agent just knows his type $P_{i}$, and reasons the probability of opponent type $P_{-i}$, but he cannot exactly know $P_{-i}$. In this case, game agent can only obtain Bayesian Nash equilibrium by the Bayesian formula. (III) For incomplete information dynamic game, at least one agent does not know the type.

If based on game agent's knowledge space, and its relationship with common knowledge, we can make the following analysis: game agent's knowledge space roughly consists of the factual propositions (notation: $\varphi$ ), public knowledge (notation: $E_{G} \varphi$ ), common knowledge (notation: $C_{G} \varphi$ ), and implicit knowledge (notation: $I_{G} \varphi$ ). The clear expression is as the following figure 2 :

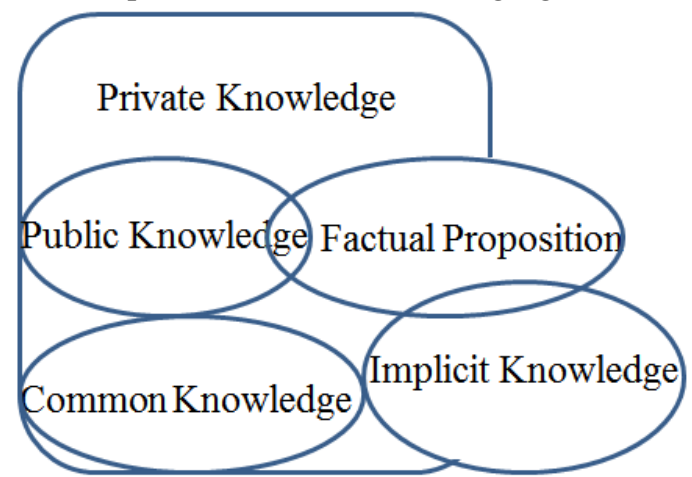

Figure 2. The relationship of private knowledge with other knowledges

\section{Game behavioral agent's knowledge structure and its formation}

As above, it is after Aumann's study about common knowledge that researcher begin to use common knowledge to analyze some hard problems. But in many cases, the research of game theory remains at information level. Information and knowledge are often lumped together or mixed up, and they don't distinguish. However, epistemic logic needs a clear cognitive concept. Therefore, it is necessary and significant to distinguish information and knowledge. The following retrospect can be the way of formation of knowledge. 


\subsection{The relationship among data, information and knowledge}

There are different among date, information and knowledge, although we usually take information as knowledge.

\subsubsection{From data to information}

Data, originating from the characterization of human, is generally believed to a sign of objective reality. Data is an unprocessed material which can show the objective existence of things and be perceived by agent, such as graphic symbol, number, letter, sound and so on $[12,13]$. Before extracted, what showed up are messy symbols. They are original materials and carriers of information and knowledge. In the game behavior, data can be any symbol related to game-agent and external space between game-agents, such as space and time symbol of game behavior. They are disorganized discrete status distribution symbols without rules.

Although information has different interpretations in different fields,it must reflect essential attribute of information.It is clear that information comes from data.Data can be said to be a kind of objective existence. Data becomes information because it involves cognitive function of game-agent.As we all know,"manufacturer A, 2015,3000 " are data but not associated.Only when they are used to describe objective reality and their relationship and form ordered data flow,can data be called information.Information is not simply the stack of data,because the result of casual stack of data is likely to be a kind of data.So information is distinguished from data in the fact that information is ordered association of data and information has timeliness.For example,in incomplete information static game, manufacturer B gets data flow "manufacturer A, 2015, 3000"from outside world. Manufacturer B can process them into:(a) Single commodity cost of manufacturer B is 3000 in 2015,(b) Monthly output of manufacturer B is 3000 in 2015, and so on.

\subsubsection{From information to knowledge}

In game theory, information is a very important concept. Information economics, the most compelling part of modern economics, is based on game theory. According to the specific needs, game agent can get information of itself by extracting, marshaling and translating data with priori knowledge and cognitive function. Ultimately, only the information which conforms to "definition 1" can be the behavioral agent's knowledge. In other words,only the knowledge perceived and confirmed by a game agent can become the behavioral agent's knowledge.

\subsubsection{Fromprivate knowledge to common knowledge}

In game behavior, the process from private knowledge to common knowledge of game agents involves game agent's belief update process by public announcements. The logic of public announcement is first proposed by logician Plaza in 1989 [14].He studied the impact of belief update on behavioral agent in detail. Moreover, a reasoning system for transforming from private knowledge to common knowledge can be referred to the literature [15].

\section{2 knowledge structure of game agent}

First, agent extracts the data from the external environment, and then transforms information to private knowledge. In the end,game agent adopts suitable strategy according to private knowledge. For this, we can construct a model figure 3 as follows:

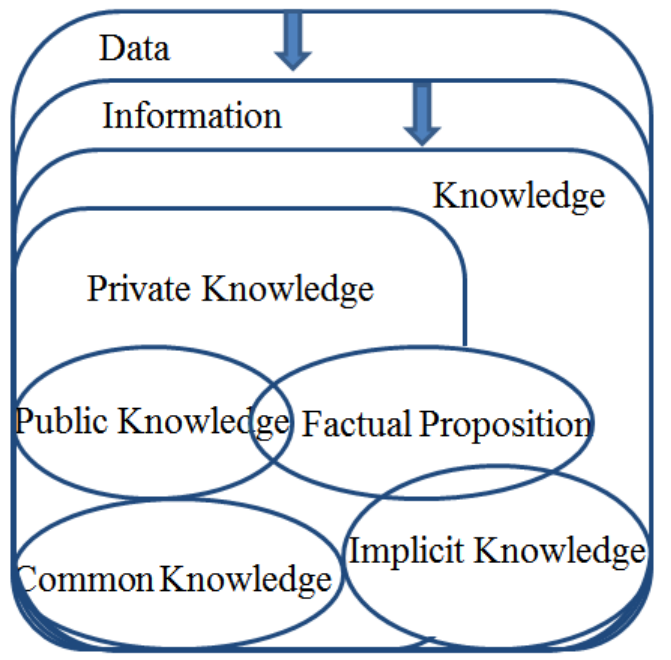

Figure 3. Agent's knowledge structure and its formation

In this model,we can clearly see the transform relationship and containment relationship among information,data,knowledge, individual knowledge and common knowledge.In the process of forming knowledge, incomplete information appeared when relevant part of information is not shared by all the agents.This often happens when some(or all)agents exclusively know their payoff function.

\section{Conclusion}

It can be found that the research about the knowledge of game agent is different from the analysis of knowledge in other fields.Because game behavior has the property of information economy,the analysis of game behavior inevitably contains the analysis tools of information economics.And then the research analyzes it gradually by epistemic logic. Colligating knowledge of different agents,reflecting the features of game behavior,this research is believed to be more scientific.On the basis of distinction of data,information,knowledge,common knowledge and private knowledge involved in game behavior,the transform process is analyzed and knowledge structure model of game agent is constructed eventually.This paper can see clearly the form of knowledge in game behavior, andobtain the essence of game agent'sknowledge. It's worth emphasizing that the analysis about the form of knowledge is a framework and cognitive game behavior needsfurther research. 


\section{Acknowledgements}

This work was supported in part by the National Natural Science Foundation of China under Grant Nos.61273338, and in part by the Major Program of National Social Science Foundation of China under Grant 11\&ZD088.

\section{References}

1. J. Hintikka,Knowledge and Belief(Cornell University Press, 1962)

2. H. H. Nax, B. S. Pradelski, Evolutionary dynamics and equitable core selection in assignment games. International Journal of Game Theory,44(4), 903-932(2015)

3. P. I. Cowling, S. Devlin, E. J. Powley, D. Whitehouse, J. Rollason, Player preference and style in a leading mobile card game.Computational Intelligence and $\mathrm{AI}$ in Games, IEEE Transactions on, 7(3), 233-242(2015)

4. J. van Benthem, Extensive Games as Process Models. Journal of logic, Language and Information, 11(3):289-313 (2002)

5. E. L.Gettier, Is justified true belief knowledge?. analysis, 23(6), 121-123(1963)

6. J.Dancy, E.Sosa,M. Steup, $A$ companion to epistemology(John Wiley \& Sons, 27-119, 2009)

7. D.Lewis, Conventions: A Philosophical Study(Harvard University Press, 1969)

8. R. J.Aumann, Agreeing to disagree. The annals of statistics, 1236-1239(1976)

9. J. Y.Halpern, Y.Moses, Knowledge and common knowledge in a distributed environment. Journal of the ACM (JACM), 37(3), 549-587(1990)

10. M. J.Osborne, A.Rubinstein, A course in game theory (MIT press, 67-84, 1994)

11. A. K.Dixit, S.Skeath, Games of Strategy: Fourth International Student Edition(WW Norton \& Company, 2015)

12. M.Frické,Big data and its epistemology. Journal of the Association for Information Science and Technology, 66(4), 651-661(2015)

13. S.Yin, X.Li, H.Gao, O.Kaynak,Data-based techniques focused on modern industry: an overview. Industrial Electronics, IEEE Transactions on, 62(1), 657-667(2015)

14. J.Plaza, Logics of public communications. Synthese, 158(2), 165-179(2007).

15. J.Gao, C.Zhou, A reasoning system about knowledge acquisition in bi-agent interaction.2016 Eighth International Conference on Advanced Computational Intelligence (ICACI). IEEE, 412-417 (2016) 